\title{
IFITM1 targets HIV-1 latently infected cells for antibody-dependent cytolysis
}

\author{
Rui André Saraiva Raposo, Miguel de Mulder Rougvie, Dominic Paquin-Proulx, Phillip M. Brailey, \\ Vinicius D. Cabido, Paul M. Zdinak, Allison S. Thomas, Szu-han Huang, Greta A. Beckerle, \\ Richard B. Jones, and Douglas F. Nixon
}

Department of Microbiology, Immunology, and Tropical Medicine, The George Washington University, Washington, DC, USA

HIV-1 persistence in latent reservoirs during antiretroviral therapy (ART) is the main obstacle to virus eradication. To date, there is no marker that adequately identifies latently infected CD4+ $T$ cells in vivo. Using a well-established ex vivo model, we generated latently infected CD4 ${ }^{+} \mathrm{T}$ cells and identified interferon-induced transmembrane protein 1 (IFITM1), a transmembrane antiviral factor, as being overexpressed in latently infected cells. By targeting IFITM1, we showed the efficient and specific killing of a latently infected cell line and CD4+ $\mathrm{T}$ cells from ART-suppressed patients through antibody-dependent cytolysis. We hypothesize that IFITM1 could mark natural reservoirs, identifying an immune target for killing of latently infected cells. These novel insights could be explored to develop clinical therapeutic approaches to effectively eradicate HIV-1.

Conflict of interest: The authors have declared that no conflict of interest exists.

Submitted: December 1, 2015 Accepted: November 17, 2016 Published: January 12, 2017

Reference information: JCI Insight. 2017;2(1):e85811. doi:10.1172/jci.insight.85811

\section{Introduction}

Antiretroviral therapy (ART) potently suppresses HIV-1, but viral eradication remains unachievable due to the persistence of reservoirs (1), which is thought to be caused by intrinsic stability (2) and homeostatic proliferation of latent cells (3); reseeding of newly infected cells by low levels of replicating HIV-1 in lymph nodes and gut-associated lymphoid tissue $(\operatorname{GALT})(4,5)$; and incomplete penetration of antiretroviral drugs into infected tissues (6). HIV-1 may continue to replicate and traffic in lymphoid tissue despite potent ART (7). Viral DNA persists in central memory (3) and stem central memory CD4 ${ }^{+} \mathrm{T}$ cells (8). Current eradication strategies rely on histone deacetylase inhibitors (HDACi) and related compounds, in conjugation with ART, to reactivate and kill infected cells within the reservoir $(9,10)$.

Components of intrinsic immunity, such as restriction factors, mediate potent anti-HIV-1 activities (11) by interfering with the virus life cycle. Restriction factor expression varies between cells and activation states (12), and in addition to acting directly against HIV-1, restriction factors promote immune responses to accelerate the clearance of infected cells. Such roles have been described for BST2/tetherin and TRIM5 (13-15). Type I interferons, such as IFN- $\alpha$, are able to strongly induce the expression of most, but not all, restriction factors to further curb recurrent cycles of infection (16). Cell-specific intrinsic immune mechanisms that orchestrate the interferon response are likely to play a key role in the prevention of HIV-1 infection in long-lived memory $\mathrm{CD}^{+} \mathrm{T}$ cell subsets.

Interferon-induced transmembrane protein 1 (IFITM1), a 14-kDa protein implicated in cell adhesion and cell growth control, has potent anti-HIV-1 activity. It is a membrane-associated protein with its C-terminus in the extracellular space and a conserved intracellular region (17). IFITM1 contains an interferon-sensitive response element in its promoter region, so it is strongly induced by IFN- $\alpha$ (18). The precise mechanism by which IFITM1 restricts HIV-1 infection is unclear. Lu et al. suggested that IFITM1 is involved in the endocytosis of HIV-1 and it may eliminate viral particles before efficient infection is established (19). IFITM1 is noticeably strong at restricting HIV-1 replication by interfering with Gag expression (19). No HIV-1 antagonist has been reported to counteract IFITM1, and no role for IFITM1 has been described during HIV-1 latency.

Latency models have predominantly been used as means to study HIV-1 reactivation in hopes of providing data toward "shock-and-kill" strategies (20). We utilized latency models to obtain unique mechanistic insights into the role of intrinsic cellular immunity during ART. Previous work by our group led to the development of a quantitative PCR-based (qPCR-based) array capable of examining the expression of a predefined set of antiviral genes in primary cells $(12,21)$. In this work, we hypothesized that transcriptional 
and epigenetic patterns found in latent and reactivated $\mathrm{CD}^{+} \mathrm{T}$ cells lead to a differential expression of restriction factors and other antiviral genes. To test this hypothesis, we generated latently infected $\mathrm{CD} 4^{+} \mathrm{T}$ cells and found IFITM1 to be overexpressed in resting latent cells when compared with their reactivated counterparts. We observed efficient and specific killing of latently infected cells through IFITM1 antibodydependent cell-mediated cytolysis (ADCC). Our finding therefore suggests that IFITM1 could potentially be exploited as a biomarker of latency, providing targets for the immunological clearance of HIV-1 reservoirs in patients.

\section{Results}

To study the dynamics of intrinsic cellular immune factors during latent infection, we generated primary HIV-1 latent cells using an ex vivo model (Figure 1A; refs. 20, 22, 23) with the HIV-1 molecular clone $\mathrm{HIV}-1_{\mathrm{NL} 4-3}$. We isolated $\mathrm{CD} 4^{+} \mathrm{T}$ cells from healthy donors, conditioned them for 3 days in the presence of CCL19, and infected them with HIV-1. We confirmed the absence of HIV-1 Gag protein by flow cytometry (KC57 antibody) (Figure 1B) at day 6 after infection in resting (CD25-, CD69-, and HLA$\mathrm{DR}^{-}$) latent $\mathrm{CD}^{+} \mathrm{T}$ cells. Latent HIV-1 was then reactivated in resting cells with anti-CD3/CD28 for 3 days, leading to a 10-fold induction in HIV-1 gag and HIV-1 mRNA transcripts, including unspliced (US), single-spliced (SS), and multiple-spliced (MS) mRNA, as measured by real-time qPCR (Figure 1C). We comprehensively analyzed the expression of selected antiviral genes and host restriction factors and found that the expression of IFITM1 and SAMHD1 was increased 4-fold and 2-fold, respectively, in resting latent cells when compared with reactivated cells (Figure 1D). IFITM1 expression was increased more than 2-fold in the latently infected ACH-2 T cell line prior to reactivation with the HDACi romidepsin (Supplemental Figure 1; supplemental material available online with this article; https:// doi.org/10.1172/jci.insight.85811DS1). We further confirmed that the frequency of IFITM1 $1^{+} \mathrm{CD} 4^{+} \mathrm{T}$ cells was reduced upon HIV-1 reactivation (Figure 1E).

Because IFITM1 is a transmembrane protein, we tested the capacity of NK cells to recognize and kill IFITM1-expressing cells by antibody-dependent cell-mediated cytotoxicity (ADCC). Latently infected $\mathrm{ACH}-2$ cells were labeled with an anti-IFITM1 antibody or control and cultured with PBMCs from healthy donors at different effector to target $(\mathrm{E} / \mathrm{T})$ ratios. Dead cells were monitored by flow cytometry using a live/dead cell marker, while the early stages of apoptosis were detected using the FAM Poly Caspases assay kit (FLICA). Representative flow plots of LIVE/DEAD Aqua versus FLICA ${ }^{+}$gated on the target cells (ACH-2) are shown in Figure 2A. In parallel, we investigated whether the cellular immune-complexes marked with anti-IFITM1 antibody and latently infected ACH-2 led to NK cell (defined as CD3 and $\mathrm{CD} 56^{+}$lymphocytes) activation, by detecting the production of intracellular IFN- $\gamma$ and degranulation $\left(\mathrm{CD} 107 \mathrm{a}^{+}\right)$. Representative flow plots are depicted in Figure 2B. We detected significant killing of $\mathrm{ACH}-2$ cells labeled with anti-IFITM1 antibody $(79 \% \pm 4.5 \%)$, when compared with control $(25 \% \pm 1.3 \%)$ (Figure $2 \mathrm{C})$, and this was associated with a significant increase $(P=0.0286)$ in the frequency of IFN- $\gamma^{+} \mathrm{CD} 107 \mathrm{a}^{+}$ NK cells $(1.345 \% \pm 1.3 \%)$ (Figure $2 \mathrm{D})$. When analyzed individually, IFN- $\gamma$ production (Figure $2 \mathrm{E}$ ) and degranulation (Figure $2 \mathrm{~F}$ ) were also significantly increased in ACH-2 cells labeled with anti-IFITM1 antibody $(P<0.05)$. These results indicate that targeting of latently infected ACH-2 cells with anti-IFITM1 leads to efficient ADCC activity and killing.

Because HIV-1 predominantly persists in a reservoir of latently infected central memory $\mathrm{CD} 4^{+} \mathrm{T}$ cells in patients on suppressive ART (3), we investigated the frequency of IFITM1+CD4 ${ }^{+} \mathrm{T}$ cells in different cell subsets (gating strategy depicted in Supplemental Figure 2). Interestingly, we found an overall increase in IFITM $1^{+} \mathrm{CD} 4^{+} \mathrm{T}$ cells in patients on ART ( $2 \%$ vs. $\left.0.9 \%\right)$, with a statistically significant increase in IFITM $1^{+} \mathrm{CD} 4^{+}$central memory $\mathrm{T}$ cells $(P=0.0260)$ (Figure 3A). To assess whether IFITM $1^{+} \mathrm{CD} 4^{+} \mathrm{T}$ cells could be killed by ADCC, we sorted $\mathrm{CD} 4^{+} \mathrm{T}$ cells from ART-suppressed patients, labeled them with anti-IFITM1 antibody, and cultured them with effector cells from the same patient for 5 hours. IFITM1 ${ }^{+} \mathrm{T}$ cells were found almost exclusively in the fraction positive for amine-reactive live/ dead marker and FLICA (Figure 3B), suggesting efficient and specific killing. To determine whether effector cells were able to produce intracellular IFN- $\gamma$ and degranulate in the presence of anti-IFITM1 antibody, we gated on NK cells and found that autologous effector cells failed to do so despite efficient killing of target cells when compared with controls (Figure 3C). We then performed the same experiments with heterologous effector cells from healthy donors, and overall found a significant production of IFN- $\gamma$ and upregulation of CD107a $(P=0.0313)$ (Figure 3C). 

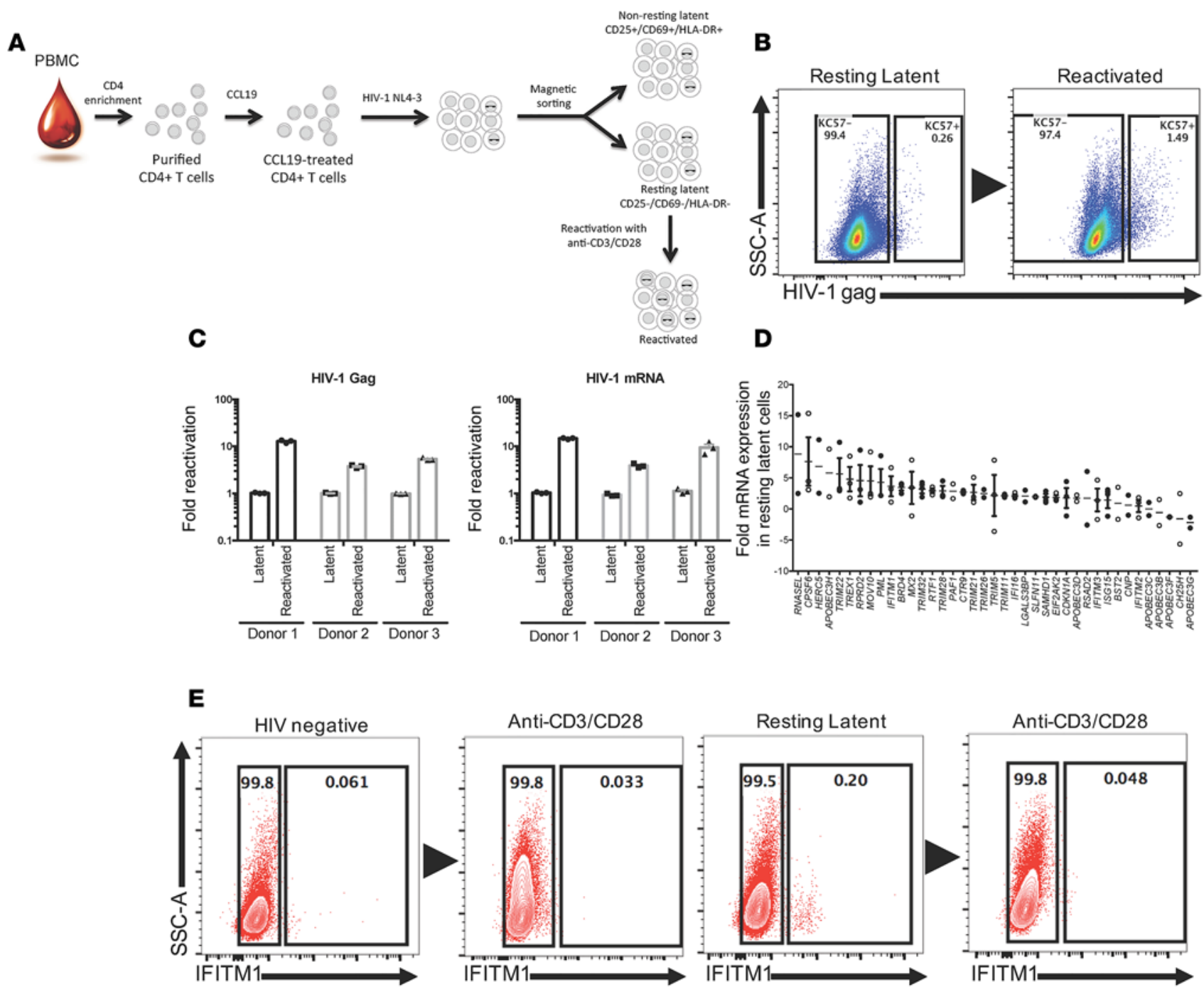

Figure 1. Generation of latently infected CD4 ${ }^{+} \mathbf{T}$ cells. (A) $\mathrm{CD} 4^{+} \mathrm{T}$ cells were negatively selected from PBMCs and conditioned for 3 days with CCL19. CCL19-treated cells were infected with HIV- $1_{\text {NL-3 }}$ for 6 days, and resting CD4+ ${ }^{+}$cells (CD25-CD69-HLA-DR-) were negatively selected by magnetic cell sorting. Resting CD4+ T cells were reactivated with anti-CD3/CD28 for 3 days. (B) Representative intracellular staining of HIV-1 Gag (KC57) ( $n=3$ ). (C) Quantification of HIV-1 gag and mRNA transcripts by real-time qPCR. (D) Fold expression of selected antiviral genes in resting latent relative to reactivated cells. Data are plotted as mean \pm SEM where $n=3$. (E) Representative extracellular staining of IFITM1 $(n=3)$.

\section{Discussion}

The use of antibody-mediated cell killing to effectively control HIV infection has gained significant interest in recent years. Recently, two papers described the use of dual-affinity re-targeting (DART) proteins aimed at improving T cell-mediated clearance of HIV-1-infected cells $(24,25)$. DARTs were specifically designed to recognize Env-expressing cells and to engage to CD3. Our approach has the advantage of targeting the HIV-1 reservoir without the need for T cell activation. Furthermore, because we are targeting a host factor, it is not possible for HIV-1 to escape killing by mutating.

Early after infection and before an efficient HIV-specific adaptive immune response is raised, NK cells are activated and contribute to the initial control of HIV-1 replication (26). In our study, despite being able to kill target cells, autologous NK cells from ART-suppressed HIV-1 patients showed limited capacity to produce IFN- $\gamma$ and to upregulate CD107a in the presence of anti-IFITM1 antibody. It will be of interest to determine whether the function of NK cells against an anti-IFITM1 antibody can be enhanced by the use of IL-15 superagonists (27).

Central memory $\mathrm{T}\left(\mathrm{T}_{\mathrm{CM}}\right)$ cells are the main source of latent virus in natural reservoirs. We detected a higher proportion of IFITM1 ${ }^{+} \mathrm{T}_{\mathrm{CM}}$ cells in ART-suppressed patients, but this does not prove that IFITM1 ${ }^{+}$ 
A
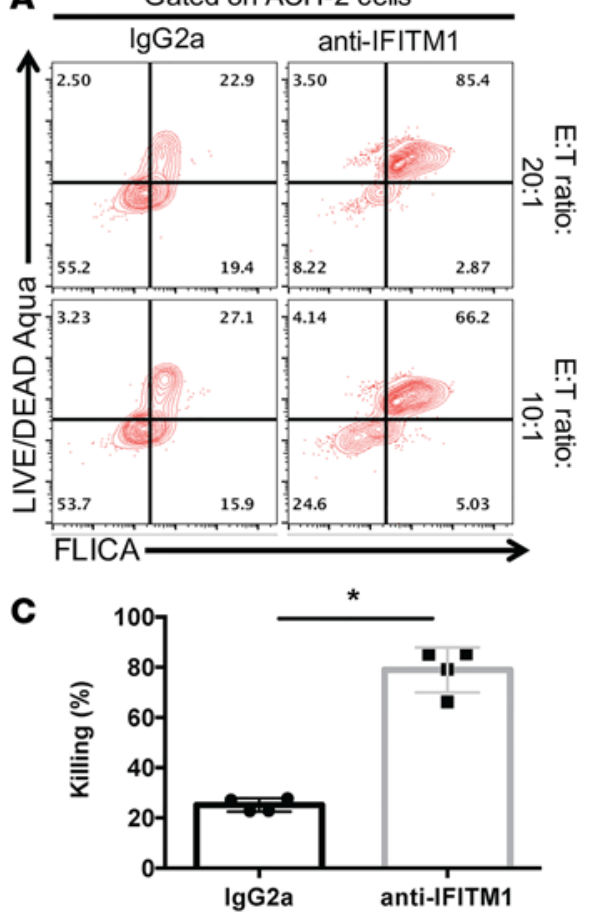

$\mathbf{E}$

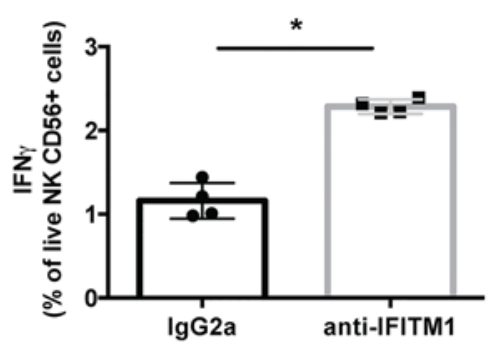

B

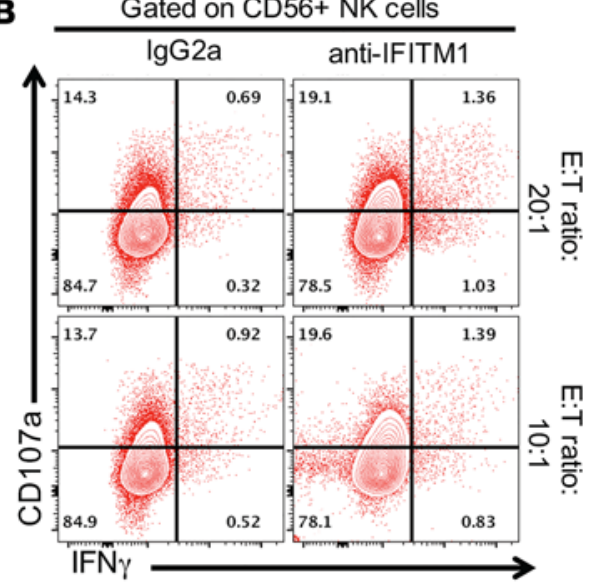

D

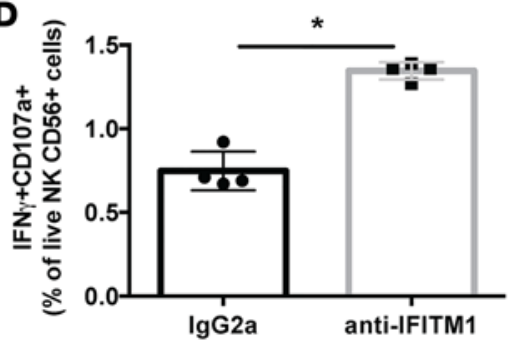

$\mathbf{F}$

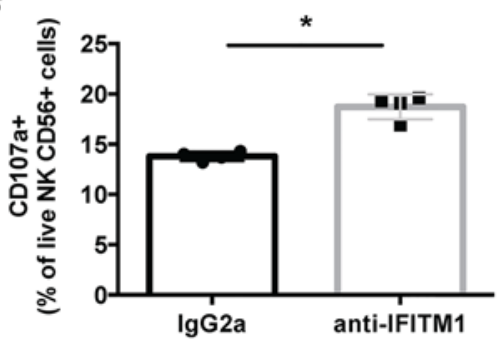

Figure 2. ADCC of latently infected ACH-2 cells. (A) ACH-2 cells (target) were incubated with anti-IFITM1 or isotype control prior to the addition of effector cells. Target cell apoptosis was detected using the FLICA assay and a live/dead marker. Representative apoptosis plot gated on $\mathrm{ACH}-2$ $(n=4)$. (B) At the end of the assay, cells were washed and stained with antibodies to detect NK cell degranulation and production of intracellular IFN- $\gamma$. Representative functional assay gated on $C D 56^{+}$NK cells at different $\mathrm{E} / \mathrm{T}$ ratios $(n=4)$. (C) Overall percentage of killed (dead/FLICA ${ }^{+}$) ACH-2 target cells; (D) IFN $-\gamma^{+}$CD107a ${ }^{+}$NK cells; (E) IFN- $\gamma^{+} \mathrm{NK}$ cells; and (F) CD107a $\mathrm{a}^{+} \mathrm{NK}$ cells $(n=4)$. Data are plotted as mean $\pm \mathrm{SD}$, and significance was determined using a nonparametric 2 -tailed $t$ test. ${ }^{*} P<0.05$.

T cells exclusively harbor latent reservoir. Further investigations are needed to determine whether the virus present in IFITM $^{+}$cells is replication competent.

We acknowledge that residual IFITM1 expression in non-latently infected cells may lead to the death of bystander cells in the event this strategy translates into an eradication approach in humans. Nonetheless, we believe that an eradication strategy involving IFITM1 in conjugation with immune checkpoint molecules shown to contribute to HIV persistence during ART (28) would be of significant value to effectively target and eliminate latently infected cells in vivo.

Three of the 5 human IFITM proteins have activity against enveloped viruses, such as influenza, West Nile virus, Dengue, hepatitis virus, and HIV. Our current understanding of the mechanism of action by which IFITM1 blocks HIV-1 replication is incomplete. Therefore, our findings also suggest the need for further studies aimed at identifying new antiviral roles for IFITM1, particularly during ART.

In sum, our results suggest a role for IFITM1 during HIV-1 latency. Importantly, the efficient killing of latently infected cells through the engagement of an anti-IFITM1 antibody may shed light into new strategies for the efficient eradication of latently infected cells.

\section{Methods}

Generation of HIV-1 latently infected $C D 4^{+} T$ cells. Latently infected $\mathrm{CD} 4^{+} \mathrm{T}$ cells were generated using the model previously described by the Lewin group (22), with some modifications. PBMCs from healthy individuals were isolated from buffy coats, and $\mathrm{CD}^{+} \mathrm{T}$ cell were isolated by negative selection (StemCell Technologies, Human CD4 ${ }^{+} \mathrm{T}$ cell Enrichment Kit, 19052). Purified CD4 ${ }^{+} \mathrm{T}$ cells were incubated with CCL19 (29 nM, R\&D Systems) for 3 days before HIV-1 infection. Cells were infected with HIV-1 ${ }_{\mathrm{NL} 4-3}$ for 4 hours at $37^{\circ} \mathrm{C}$. The cells were then cultured in the presence of IL-2 (10 IU/ml, Roche) for 6 days. At day 6 after infection, resting CD4 ${ }^{+} \mathrm{T}$ cells were isolated by anti-PE magnetic bead depletion (Miltenyi Biotec, Anti-PE Microbeads, 130-048-801) using a cocktail of antibodies to CD25-PE (BD Pharmingen, clone M-A251), CD69-PE (BD Pharmingen, clone FN50), and HLA-DR-PE (BD Pharmingen, clone G46-6). Resting latent $\mathrm{CD}^{+} \mathrm{T}$ cells were then reactivated with anti-CD3/CD28 (StemCell Technologies) for 3 days in the presence of IL-2 $(50 \mathrm{IU} / \mathrm{ml})$, according to the manufacturer's specifications. 

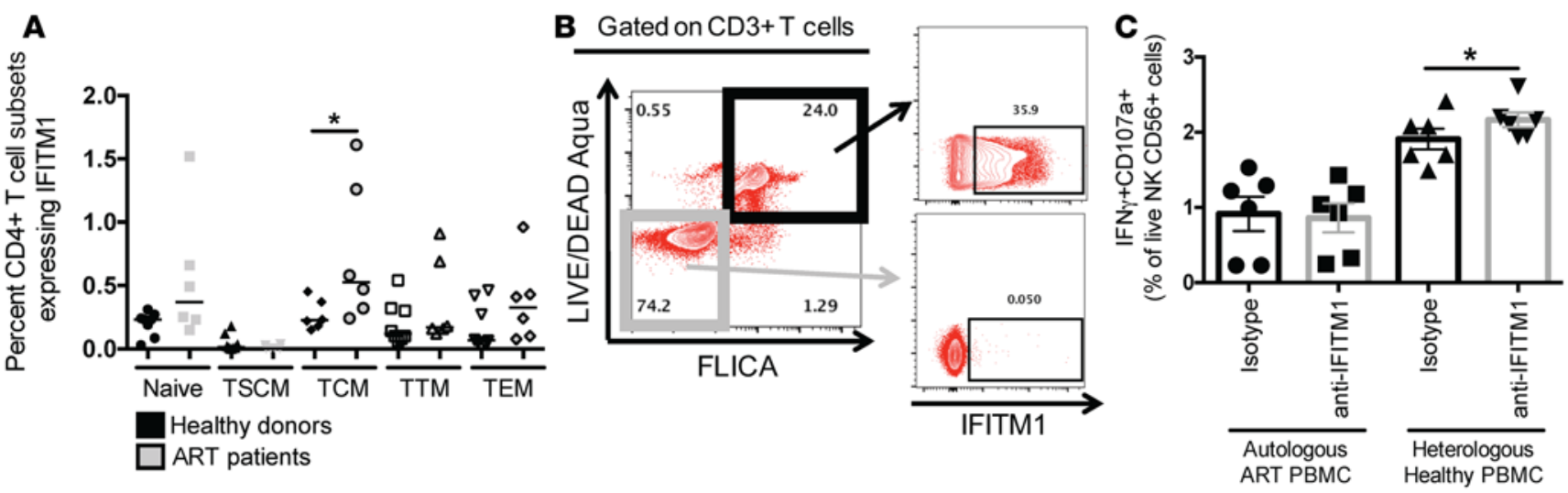

Figure 3. ADCC of IFITM1+CD4+ T cells from ART-suppressed patients. (A) Expression of IFITM1 in CD4 ${ }^{+}$T cell subsets from healthy $(n=6)$ and ART-suppressed patients $(n=6)$. Naive, stem central memory (TSCM), central memory (TCM), transitional memory (TTM), and effector memory (TEM). (B) Purified CD4+ T cells from ART-suppressed patients were incubated with anti-IFITM1 or isotype control prior to the addition of autologous effector cells at an E/T ratio of 10:1. Target cell apoptosis was detected using the FLICA assay and a live/dead marker $(n=6)$. (C) Cells were washed and stained with anti-CD3, -CD56, -CD107a, and -IFN- $\gamma$ to detect NK cell degranulation and production of intracellular IFN- $\gamma$. Overall percentage of IFN- $\gamma^{+}$CD107a ${ }^{+}$NK cells in autologous or heterologous conditions $(n=6)$. Data are plotted as mean \pm SD, and significance was determined using a nonparametric 2-tailed $t$ test. ${ }^{*} P<0.05$.

ACH-2 cell line and reactivation with histone deacetylase inhibitors. ACH-2 cells were obtained from the NIH AIDS Reagents Program (catalog 349) and were tested negative for mycoplasma contamination. Cells were typically propagated in RPMI1640, 2 mM L-glutamine, 10\% FBS in the presence of penicillin/streptomycin. Vorinostat (SAHA), panobinostat, and romidepsin were purchased from Selleck Chemicals. Cells were typically plated in 96-well plates (U-shaped) and treated with different concentrations of HDACi for 5 hours, washed twice, and recultured for 18 hours prior to RNA extraction or flow cytometry.

Flow cytometry. To detect intracellular HIV-1 Gag, CD4 ${ }^{+} \mathrm{T}$ cells were washed in FACS buffer $(2 \mathrm{mM}$ EDTA, $0.5 \%$ BSA in ice-cold PBS) and surface stained with antibodies against CD3 (1:100, ECD, clone UCHT1, Beckman Coulter), CD4 (1:500, Alexa Fluor 700, clone RPA-T4, Becton Dickinson), CD8 (1:400, BV605, clone RPA-T8, BioLegend), and a live/dead marker (1:200, Invitrogen) emitting in the aqua wavelength, for 30 minutes at $4^{\circ} \mathrm{C}$. After staining, the cells were washed 3 times, fixed in $2 \%$ paraformaldehyde for 20 minutes, and stained with KC57-RD1 antibody (1:100, Beckman Coulter) in $0.1 \%$ saponin for 30 minutes. For the detection of IFITM1 in different CD4 ${ }^{+} \mathrm{T}$ cell subsets, PBMCs were washed in FACS buffer and surface stained with monoclonal anti-IFITM1 antibody (1:200, 60074-1-Ig, Proteintech) for 30 minutes at $4^{\circ} \mathrm{C}$. After staining, cells were washed 3 times and stained with anti-mouse IgG2aPE (1:200, BioLegend). Following IFITM1 staining, PBMCs were washed and stained for CD3 (1:100, ECD, clone UCHT1, Beckman Coulter), CD4 (1:500, Alexa Fluor 700, clone RPA-T4, Becton Dickinson), CD8 (1:400, BV605, clone RPA-T8, BioLegend), CD45RA (1:100, BV650, clone HI100, BD Pharmingen), CCR7 (1:100, PE-Cy7, clone G043H7, BioLegend), CD27 (1:100, APC-Cy7, clone 0323, BioLegend), and CD95 (1:50, Pacific Blue, clone DX2, BioLegend) and a live/dead marker (1:200, Invitrogen) for $30 \mathrm{~min}$ utes at $4^{\circ} \mathrm{C}$. Fluorescence minus one controls (FMOs) and single-stained Polystyrene beads (BD Biosciences) were conducted for compensation purposes, and the gating strategy is shown in Supplemental Figure 2. Cells were washed and events were collected on an LSRFortessa X-20 flow cytometer (BD Biosciences). Data were analyzed using FlowJo software (version X.0.4, TreeStar).

ADCC and functional assays. ACH-2 cells (NIH AIDS Reagents Program) or $\mathrm{CD}^{+} \mathrm{T}$ cells, isolated from PBMCs of ART-suppressed patients by negative selection (StemCell Technologies), were incubated with monoclonal anti-IFITM1 antibody (1:200, 60074-1-Ig, Proteintech) or IgG2a isotype control (BioLegend) prior to the addition of PBMC effector cells for 5 hours at the indicated E/T ratio (20:1 and 10:1, in a 96-well U-shaped plate in a final volume of $200 \mu \mathrm{l}$ ). Autologous PBMCs or heterologous PBMCs from healthy donors were used as effector cells. Monensin (Golgi Stop, BD Biosciences) was added at the beginning of the assay. Target cell apoptosis was detected using the fluorescent inhibitor of caspases (FLICA) flow cytometry-based assay as described by Leeansyah et al. (29). Briefly, the FLICA reagent (Vybrant FAM Poly Caspases Assay Kit, ThermoFisher) was added in the last hour to the cell culture media. When the assay was terminated, cells were washed and stained with a secondary antibody against 
IFITM1 (anti-mouse IgG2a-PE, 1:200, BioLegend), washed, and surface stained for CD3 (1:100, ECD, clone UCHT1, Beckman Coulter), CD56 (1:20, PE-Cy7, clone MEM-188, BioLegend), CD107a (1:10, APC-H7, clone H4A3, BD Biosciences), and a live/dead marker (1:200, Invitrogen) for 30 minutes at $4^{\circ} \mathrm{C}$. After staining, cells were washed and fixed in Cytofix/Cytoperm for 20 minutes at $4^{\circ} \mathrm{C}$. Intracellular staining was performed using anti-IFN- $\gamma$ antibody (1:10, APC, clone B27, BD Biosciences) in Perm/ Wash (BD Biosciences). Samples were acquired on an LSRFortessa X-20 flow cytometer (BD Biosciences). Single-stained Polystyrene beads (BD Biosciences) were used for compensation purposes. Data were analyzed using FlowJo software (version X.0.4, TreeStar).

Quantification of antiviral gene expression. Total RNA from $\mathrm{CD}^{+} \mathrm{T}$ cells was extracted using TRIzol reagent, followed by RNA Cleanup with in-solution DNAaseI treatment option using QIAGEN RNaseFree DNase Set. DNase-treated clean RNA was transcribed into cDNA using random primers and the SuperScript VILO cDNA Synthesis Kit (Invitrogen), according to manufacturer's instructions. Quantitative real-time PCR on blinded samples utilized custom-made TaqMan Low Density Array (TLDA) from Applied Biosystems, and gene cards used for quantification were first described in ref. 12. Thermal cycling was performed using an ABI ViiA 7 Real-Time PCR System. Complementary DNA (cDNA) in $100 \mu$ Applied Biosystems TaqMan Universal PCR Master Mix, with UNG was loaded onto the designated ports of the TLDA plates. Data were analyzed using ABI ViiA 7 software. A panel of 6 housekeeping genes was included in the TLDA plates (GAPDH, 18S, ACTB, PPIA, RPLPO, and UBC). UBC was identified as the most stably expressed gene from those 6 housekeeping genes among the whole samples using the geNorm algorithm (30). Raw cycle threshold numbers of amplified gene products were normalized to the housekeeping gene, $U B C$ (ubiquitin $\mathrm{C}$ ), to control for cDNA input amounts.

Quantification of HIV-1 RNA transcripts by real-time qPCR. HIV-1 gag and mRNA transcripts were detected using previously described primers and probes (9, 31, 32). CCR5 (Life Technologies, Hs99999149_s1) was used as endogenous control. Real-time qPCR was performed in duplicate using TaqMan Universal PCR Master Mix (Applied Biosystems) on an ABI ViiA 7 Real-Time PCR machine. Fold induction was determined using the delta delta Ct method.

Statistics. Statistical comparisons were performed using nonparametric 2-tailed $t$ tests in GraphPad Prism. Data are plotted as mean \pm SEM or SD and $P$ values less than 0.05 were considered significant.

Study approval. Leukapheresis samples were obtained from HIV-1-infected individuals with undetectable plasma viremia ( $<50$ copies $/ \mathrm{ml}$ ) on stable ART. Individuals were recruited from the Maple Leaf Medical Clinic in Toronto, Ontario, Canada, through a protocol approved by the University of Toronto Institutional Review Board. Secondary use of the samples from Toronto was approved through the George Washington University Institutional Review Board. Anonymous PBMCs from HIV-1-seronegative individuals were obtained from the New York Blood Center. All subjects were at least 21 years of age at the time of sample collections and provided written informed consent for study participation. This study adheres to the Declaration of Helsinki Principles.

\section{Author contributions}

RASR, MMR, DPP, RBJ, and DFN conceived and designed the experiments. RASR, MMR, DPP, PMB, VDC, AST, PMZ, SH, and GAB performed the experiments. RASR, MMR, DPP, RBJ, and DFN analyzed the data. AST, SH, and RBJ contributed reagents/materials. RASR, MMR, DPP, RBJ, and DFN wrote the manuscript.

\section{Acknowledgments}

This work was supported by NIH grants (AI093179, AI076059, 1UM1AI26617) to DFN; the Peter and Shelagh Godsoe Family Foundation thorough the AIDS Research Institute at UCSF (to DFN); and the District of Columbia Center for AIDS Research (DC D-CFAR) (P30-AI087714 and P30-AI117970 to RASR and DFN). The authors thank Claudia Brockmeyer and Henri-Alexandre Michaud for helpful discussions. The authors gratefully acknowledge reagents supplied by the NIH AIDS Research and Reference Reagent Program.

Address correspondence to: Douglas F. Nixon, Department of Microbiology, Immunology, and Tropical Medicine, The George Washington University, Ross Hall 502, 2300 Eye Street, NW, Washington, DC 20037, USA. Phone: 202.994.3532; E-mail: dnixon@gwu.edu. 
1. Chun TW, Moir S, Fauci AS. HIV reservoirs as obstacles and opportunities for an HIV cure. Nat Immunol. 2015;16(6):584-589.

2. Siliciano JD, et al. Long-term follow-up studies confirm the stability of the latent reservoir for HIV-1 in resting CD4+ T cells. Nat Med. 2003;9(6):727-728.

3. Chomont N, et al. HIV reservoir size and persistence are driven by T cell survival and homeostatic proliferation. Nat Med. 2009;15(8):893-900.

4. Chun TW, et al. HIV-infected individuals receiving effective antiviral therapy for extended periods of time continually replenish their viral reservoir. J Clin Invest. 2005;115(11):3250-3255.

5. Veazey RS, Lackner AA. Getting to the guts of HIV pathogenesis. J Exp Med. 2004;200(6):697-700.

6. Fletcher CV, et al. Persistent HIV-1 replication is associated with lower antiretroviral drug concentrations in lymphatic tissues. Proc Natl Acad Sci USA. 2014;111(6):2307-2312.

7. Lorenzo-Redondo R, et al. Persistent HIV-1 replication maintains the tissue reservoir during therapy. Nature. 2016;530(7588):51-56.

8. Buzon MJ, et al. Long-term antiretroviral treatment initiated at primary HIV-1 infection affects the size, composition, and decay kinetics of the reservoir of HIV-1-infected CD4 T cells. J Virol. 2014;88(17):10056-10065.

9. Archin NM, et al. Administration of vorinostat disrupts HIV-1 latency in patients on antiretroviral therapy. Nature. 2012;487(7408):482-485.

10. Elliott JH, et al. Short-term administration of disulfiram for reversal of latent HIV infection: a phase 2 dose-escalation study. Lancet HIV. 2015;2(12):e520-e529.

11. Simon V, Bloch N, Landau NR. Intrinsic host restrictions to HIV-1 and mechanisms of viral escape. Nat Immunol. 2015;16(6):546-553.

12. Raposo RA, et al. Effects of cellular activation on anti-HIV-1 restriction factor expression profile in primary cells. $J$ Virol. 2013;87(21):11924-11929.

13. Galão RP, Le Tortorec A, Pickering S, Kueck T, Neil SJ. Innate sensing of HIV-1 assembly by Tetherin induces NFkB-dependent proinflammatory responses. Cell Host Microbe. 2012;12(5):633-644.

14. Pertel T, et al. TRIM5 is an innate immune sensor for the retrovirus capsid lattice. Nature. 2011;472(7343):361-365.

15. Arias JF, et al. Tetherin antagonism by Vpu protects HIV-infected cells from antibody-dependent cell-mediated cytotoxicity. Proc Natl Acad Sci USA. 2014;111(17):6425-6430.

16. Doyle T, Goujon C, Malim MH. HIV-1 and interferons: who's interfering with whom? Nat Rev Microbiol. 2015;13(7):403-413.

17. Weston S, Czieso S, White IJ, Smith SE, Kellam P, Marsh M. A membrane topology model for human interferon inducible transmembrane protein 1. PLoS One. 2014;9(8):e104341.

18. Martensen PM, Justesen J. Small ISGs coming forward. J Interferon Cytokine Res. 2004;24(1):1-19.

19. Lu J, Pan Q, Rong L, He W, Liu SL, Liang C. The IFITM proteins inhibit HIV-1 infection. J Virol. 2011;85(5):2126-2137.

20. Spina CA, et al. An in-depth comparison of latent HIV-1 reactivation in multiple cell model systems and resting CD4+ T cells from aviremic patients. PLoS Pathog. 2013;9(12):e1003834.

21. Abdel-Mohsen M, et al. Expression profile of host restriction factors in HIV-1 elite controllers. Retrovirology. 2013;10:106.

22. Saleh S, Solomon A, Wightman F, Xhilaga M, Cameron PU, Lewin SR. CCR7 ligands CCL19 and CCL21 increase permissiveness of resting memory CD4+ T cells to HIV-1 infection: a novel model of HIV-1 latency. Blood. 2007;110(13):4161-4164.

23. Saleh S, et al. Expression and reactivation of HIV in a chemokine induced model of HIV latency in primary resting CD4+ T cells. Retrovirology. 2011;8:80.

24. Sung JA, et al. Dual-Affinity Re-Targeting proteins direct T cell-mediated cytolysis of latently HIV-infected cells. J Clin Invest. 2015;125(11):4077-4090.

25. Sloan DD, et al. Targeting HIV reservoir in infected CD4 T cells by dual-affinity re-targeting molecules (DARTs) that bind HIV envelope and recruit cytotoxic T cells. PLoS Pathog. 2015;11(11):e1005233.

26. Alter G, et al. Evolution of innate and adaptive effector cell functions during acute HIV-1 infection. J Infect Dis. 2007;195(10):1452-1460.

27. Seay $\mathrm{K}$, et al. In vivo activation of human NK cells by treatment with an interleukin-15 superagonist potently inhibits acute in vivo HIV-1 infection in humanized mice. J Virol. 2015;89(12):6264-6274.

28. Fromentin R, et al. CD4+ T cells expressing PD-1, TIGIT and LAG-3 contribute to HIV persistence during ART. PLoS Pathog. 2016;12(7):e1005761.

29. Leeansyah E, et al. Arming of MAIT Cell cytolytic antimicrobial activity is induced by IL-7 and defective in HIV-1 infection. PLoS Pathog. 2015;11(8):e1005072.

30. Vandesompele J, et al. Accurate normalization of real-time quantitative RT-PCR data by geometric averaging of multiple internal control genes. Genome Biol. 2002;3(7):RESEARCH0034.

31. Shan L, et al. A novel PCR assay for quantification of HIV-1 RNA. J Virol. 2013;87(11):6521-6525

32. Bullen CK, Laird GM, Durand CM, Siliciano JD, Siliciano RF. New ex vivo approaches distinguish effective and ineffective single agents for reversing HIV-1 latency in vivo. Nat Med. 2014;20(4):425-429. 